\title{
Electrodynamics of graphene heterostructures and electromagnetic applications
}

\author{
Philippe Lambin \\ University of Namur \\ 5000 Namur, Belgium \\ philippe.lambin@unamur.be \\ Bruno Majérus \\ University of Namur \\ 5000 Namur, Belgium \\ bruno.majerus@unamur.be
}

\author{
Mirko Cormann \\ University of Namur \\ 5000 Namur, Belgium \\ mirko.cormann@unamur.be
}

\author{
Tommi Kaplas \\ University of Eastern Finland \\ 80101 Joensuu, Finland \\ tommi.kaplas@uef.fi
}

\author{
Michaël Lobet \\ J.A. Paulson School Eng. Appl. Sci. \\ Harvard University \\ Cambridge, MA 02138, USA
}

mlobet@seas.harvard.edu

\author{
Yuri Svirko \\ University of Eastern Finland \\ 80101 Joensuu, Finland \\ yuri.svirko@uef.fi \\ Polina Kuzhir \\ Belarusian State University \\ Minsk, 220030 Belarus \\ polina.kuzhir@gmail.com
}

\author{
Michaël Sarrazin \\ University of Namur \\ 5000 Namur, Belgium \\ michael.sarrazin@unamur.be
}

Alesia Paddubskaya

Belarusian State University

Minsk, 220030 Belarus

paddubskaya@gmail.com

Konstantin Batrakov

Minsk, 220030 Belarus

kgbatrakov@gmail.com

\begin{abstract}
Heterostructures combining graphene layers, dielectric slabs and even metallic substrates are interesting systems to look at in view of their potential applications in microwaves and terahertz. A short review of their properties is presented, together with possible applications such as shielding layers, polarizers and plasmonic devices. In the near infrared and visible range, a graphene layer modifies the Brewster angle of the substrate that supports it. This effect leads to a non-destructive technique to count the number of atomic planes the graphene is made of.

Index Terms-radar absorbing material, electromagnetic interference compatibility, optical conductivity
\end{abstract}

\section{INTRODUCTION}

Graphene is a genuine two-dimensional material with remarkable properties in many domain, including electromagnetism. Although infinitely-thin at the macroscopic scale, graphene does interact strongly with electromagnetic radiations. In the infra-red range and a large part of the visible spectrum, a single atomic plane of graphene absorbs $2.3 \%$ of the incident radiation at normal incidence. This fraction changes with increasing incidence angle and depends on the polarization [1]. When deposited on a flat dielectric surface, graphene modifies the Brewster angle of the air/substrate interface. This modification can be measured by ellipsometry and used for non-contact optical conductivity characterization [2].

In the $\mathrm{THz}$ domain and below, Drude conductivity starts to rule over the electronic inter-band transitions. The electromagnetic absorbance can reach almost $100 \%$ at normal incidence on monolayer graphene under special resonant conditions [3]. In the $\mathrm{GHz}$ domain, broadband absorption can be realized and adjusted almost at will. A few layers of graphene separated by ultrathin polymer sheets constitute an interesting passive device for $\mathrm{GHz}$ applications [4] Shielding layers, polarizers, and absorbing screens are among the few examples that will be described in this short review.

\section{Formalism}

It will be enough here to focus on the expressions of the reflectance $R$ and absorbance $A$ of graphene -or any conducting layer that is ultra-thin on the scale of its bulk skin depth-placed at the interface between two semi-infinite nonabsorbing media. A plane wave is impinging the conducting layer from medium 1 at an incidence angle $\theta_{1}$ and is partly transmitted in medium 2 at the refraction angle $\theta_{2}$. For the $p$ polarization under consideration here, Fresnel-like formulas write [1]

$$
\begin{aligned}
& R_{p}=\left|\frac{-n_{1} \sec \theta_{1}+n_{2} \sec \theta_{2}+\tilde{\sigma}}{n_{1} \sec \theta_{1}+n_{2} \sec \theta_{2}+\tilde{\sigma}}\right|^{2} \\
& A_{p}=\frac{4 n_{1} \sec \theta_{1} \operatorname{Re} \tilde{\sigma}}{\left|n_{1} \sec \theta_{1}+n_{2} \sec \theta_{2}+\tilde{\sigma}\right|^{2}} .
\end{aligned}
$$

From here, $n_{1}$ and $n_{2}$ are the indices of refraction of the two media and $\tilde{\sigma}$ is the sheet conductivity of the interfacial layer in units of the intrinsic vacuum admittance $\varepsilon_{0} c$, hereafter called the reduced conductivity.

Expressions of the dynamical conductivity of graphene have been derived by many authors. The simplest approach relies on the Kubo formalism, complemented by some empirical treatment of the inelastic scattering of the charge carriers described by an average collisional rate $\gamma$. The result depends on the temperature $T$, the absolute value $\mu$ of the chemical 
potential measured from the Dirac point, and the tight-binding $\pi$ hopping parameter $t$

$$
\begin{aligned}
\tilde{\sigma}= & 4 \alpha \frac{\mu+2 k_{B} T \ln \left(1+e^{-\mu / k_{B} T}\right)}{\hbar(\gamma-i \omega)} \\
+ & \alpha \pi f_{1}\left(\frac{\hbar \omega}{t}\right)\left[\tanh \left(\frac{\hbar \omega-2 \mu}{4 k_{B} T}\right)+\tanh \left(\frac{\hbar \omega+2 \mu}{4 k_{B} T}\right)\right] \mathrm{Fa}(\hbar \omega) \\
& -i \alpha f_{2}\left(\frac{\hbar \omega}{t}\right) \ln \left(\frac{|\hbar \omega+2 \mu|-2 k_{B} T \psi}{|\hbar \omega-2 \mu|+2 k_{B} T \psi}\right)
\end{aligned}
$$

with $\alpha$ the fine-structure constant and where

$$
\begin{aligned}
\psi & =\tanh \left(\frac{|\hbar \omega+2 \mu|}{4 k_{B} T}\right)-\tanh \left(\frac{|\hbar \omega-2 \mu|}{4 k_{B} T}\right) \\
\mathrm{Fa}(\hbar \omega) & =\frac{\left(\hbar \omega-E_{\pi}+q W / 2\right)^{2}}{\left[\left(\hbar \omega-E_{\pi}\right)^{2}+(W / 2)^{2}\right]}
\end{aligned}
$$

The first term in eq. 3 accounts for intra-band scattering mechanisms, the next two terms come from $\pi-\pi^{*}$ electronic transitions and includes a Fano resonance (factor $\mathrm{Fa}$ ), with width $W$ and coupling asymmetry parameter $q$, produced by excitonic corrections to the transition $\hbar \omega=2 t$ between van Hove singularities at the $M$ point of the first Brillouin zone [8]. The details of the $\pi$ and $\pi^{*}$ bands are contained in the expressions of the functions $f_{1}\left(\frac{\hbar \omega}{t}\right)$ and $f_{2}\left(\frac{\hbar \omega}{t}\right)$ [10].

For all the applications that follow, $t$ was taken from the value of the $\hbar \omega=2 t$ transition predicted at $5.20 \mathrm{eV}$ by GW calculations [8]. The other parameters for CVD graphene are listed in the Table I.

TABLE I

CONDUCTIVITY PARAMETERS AT ROOM TEMPERATURE FOR CVD GRAPHENE (SEE EQ. 3)

\begin{tabular}{|c|c|c|c|c|c|c|}
\hline$\tilde{\sigma}_{\mathbf{D C}}$ & $\gamma\left(10^{12} \mathbf{~ r a d} / \mathbf{s}\right)$ & $\mu(\mathbf{e V})$ & $\mathbf{q}$ & $\mathbf{E}_{\pi}(\mathbf{e V})$ & $\mathbf{W}(\mathbf{e V})$ & $\mathbf{R e f}$ \\
\hline 0.37 & \multirow{2}{*}{20.0} & \multirow{2}{*}{0.14} & & & & {$[5]$} \\
& & & & & & {$[6]$} \\
0.40 & & & -1.0 & 5.02 & 0.78 & {$[7]$} \\
& & & & & & \\
\hline
\end{tabular}

\section{Discussion}

The reduced conductivity of monolayer graphene computed with the parameters of Table I is plotted in Fig. 1. With this data in hand, interesting phenomena can be derived from the expressions of the transmittance and absorbance of graphene.

\section{A. Absorbing layers}

Screening electromagnetic (em) radiations is an important issue in electromagnetic interference and electromagnetic compatibility. Blocking the transmission of em radiations with a reflecting layer is not satisfactory, because it contributes to increase em pollution. The alternative is to absorb as much as possible of the impinging power, like radar absorbing materials do. A conducting layer with thickness much smaller than the skin depth can absorb a substantial fraction of em radiations [9]. This property can readily be caught from eq. 2 in the particular case of normal incidence. The expression of the absorbance then simplifies in

$$
A=\frac{4 n_{1} \operatorname{Re} \tilde{\sigma}}{\left|n_{1}+n_{2}+\tilde{\sigma}\right|^{2}}
$$

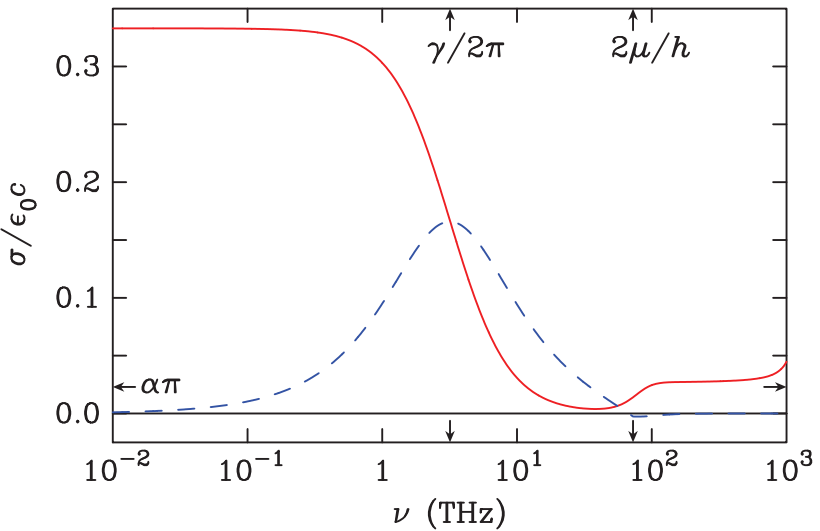

Fig. 1. Real part (solid line) and imaginary part (dotted line) of the reduced conductivity of graphene versus frequency $v=\omega / 2 \pi$ computed with eq. 3 ( $\mu$ $\left.=0.15 \mathrm{eV}, \gamma=20 \times 10^{12} \mathrm{rad} / \mathrm{s}, T=300 \mathrm{~K}\right)$.

irrespective of the polarization. It has a maximum equal to $n_{1} /\left(n_{1}+n_{2}\right)$ when $\tilde{\sigma}$ is real and equal to $n_{1}+n_{2}$, in other words when the sheet conductivity $\sigma$ matches the sum of admittance of the surrounding semi-infinite media, $n_{1} \varepsilon_{0} c+n_{2} \varepsilon_{0} c$. In this respect, graphene is a remarkable material for frequencies below $50 \mathrm{GHz}$ where $\operatorname{Re} \tilde{\sigma}$ is of the order of 0.5 while $\operatorname{Im} \tilde{\sigma} \ll \operatorname{Re} \tilde{\sigma}$ (see Fig. 1). It means that a few graphene planes suffice to realize the admittance matching condition [5]. In free air $\left(n_{1}=n_{2}=1\right)$, one needs $\tilde{\sigma}=2$, which can be obtained with 6 graphene planes that would absorb 50\% of the incoming em power for all frequencies below $50 \mathrm{GHz}$. If the transmission medium could be a metamaterial with near-zero refractive index $\left(n_{2} \approx 0\right)$, for instance an epsilon-near-zero medium, 3 graphene planes would absorb almost 100\% [11].

A simple way to mimic a near-zero-parameter medium is to resort to the Salisbury screen. Here, the graphene is supposed being held by a dielectric slab, itself deposited on a metallic plate whose thickness must be larger than its skin depth for the radiations of interest. The graphene layer is exposed to air $\left(n_{1}=1\right)$. When the thickness of the slab corresponds to a quarter wavelength, the electric field can simultaneously be zero at the interface with gold and maximum at the graphene layer, maximizing thereby the by Joule power loss. Under the assumption that the metallic plate is an ideal conductor, the absorbance of the system at normal incidence can readily be derived in the form

$$
A=\frac{4 a \operatorname{Im} \tilde{\sigma}+4 \operatorname{Re} \tilde{\sigma}}{(a+\operatorname{Im} \tilde{\sigma})^{2}+(1+\operatorname{Re} \tilde{\sigma})^{2}} \quad a=\sqrt{\epsilon} \cot \left(\sqrt{\epsilon} k_{0} d\right)
$$

with $k_{0}=\omega / c, \epsilon$ and $d$ being the permittivity and thickness of the slab. Maxima of $A$ arise when $a=0$ (quarter-wavelength case). Their amplitude is $4 \operatorname{Re} \tilde{\sigma} /|1+\tilde{\sigma}|^{2}$, which exactly corresponds to eq. 6 for $n_{2}=0$.

Producing few-layer graphene with a controlled number of atomic planes by CVD is difficult. For that reason, it is more reliable, although time-consuming, to stack individual graphene layers on top of each other. The manipulation of individual graphene planes is usually performed via a thin 
PMMA film on which graphene sticks. By piling up a few PMMA/graphene units on a substrate with suitable thickness, a heterostructure such as sketched in fig. 2 is obtained, which can reach about $75 \%$ absorbance [6]. Such a high value of the absorbance is possible when the radiations come from the back side of the supporting slab and escape from the graphene side. This geometry is perfect to shield the optical window of an optoelectronic device against microwaves. Indeed, a few layer graphene remains nearly transparent in the NIR and a large fraction of the visible spectrum, while absorbing a great part of microwaves. When the slab thickness corresponds to a quarter wavelength of the microwave to be screen, reflection is minimized. The absorbance can therefore increase to reach the ultimate value $\epsilon /(\epsilon+1)$ where $\epsilon$ is the permittivity of the slab [12].

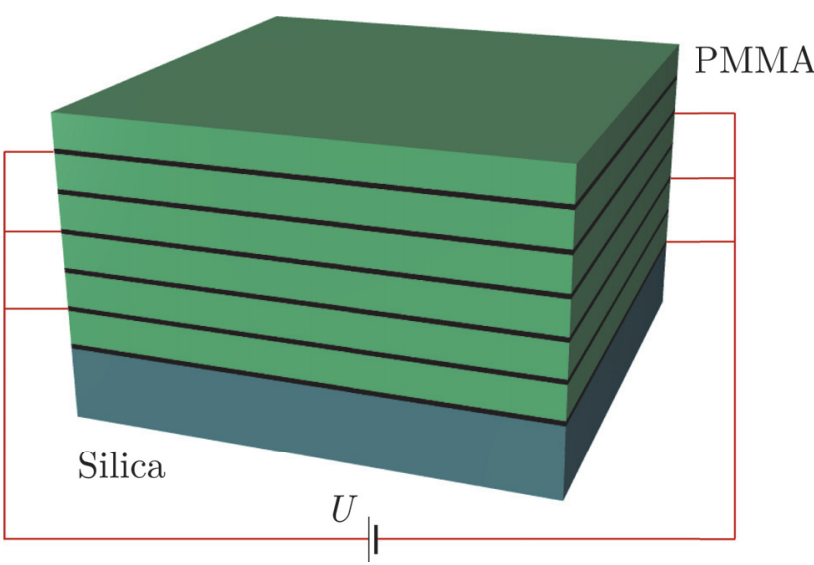

Fig. 2. Graphical representation of a heterostructure comprising a silica slab on top of which 6 graphene/PMMA units have been deposited. The PMMA spacers are a few hundred nanometer thick. The red lines schematize interdigited connections of the graphene sheets to an external power supply used to electrostatically dope the graphene sheets.

In principle, the absorbance and, hence, transmittance of the heterostructure can be tuned by modifying the graphene sheet conductivity $\sigma$ by electrostatic doping. In the device illustrated in Fig. 2 [13], the application of a static voltage $U$ increases the charge carrier density on one set of graphene planes and decreases it by the same amount on the other set. There is a net change of conductivity $\sigma(U)=$ $\sigma_{0}\left(\sqrt{\left|1+U / U_{c}\right|}+\sqrt{\left|1-U / U_{c}\right|}\right) / 2 . \sigma_{0}$ is the "intrinsic" conductivity of the graphene planes, $U_{c}=N_{0} e d / \varepsilon_{0} \epsilon$, with $N_{0}$ the "intrinsic" charge carrier density, $d$ and $\epsilon$ being the thickness and the static permittivity of the polymer spacers. $U_{c}=7.5 \mathrm{~V}$ for 200-nm thick PMMA spacers $(\epsilon=3.6)$, assuming $N_{0}$ $=0.75 \times 10^{16} \mathrm{~m}^{-2}$. The induced conductivity $\sigma(U)$ decreases with increasing $|U|$ in the interval $\left(0, U_{c}\right)$ and then raises to exceed $\sigma_{0}$ whenever $|U|>1.25 U_{c}$. As a consequence, the transmittance of the multilayer can be modified at will: it increases when $|U|$ varies between 0 and $U_{c}$ and decreases when $|U|$ exceeds $U_{c}$ [13]. The practical problem to overcome here is to avoid leakage current across the PMMA spacers and dielectric breakdown.
TABLE II

DEGREE OF POLARIZATION OF THE BEAMS REFLECTED AND TRANSMITTED BY A SELF-SUPPORTED BILAYER GRAPHENE $(\tilde{\sigma}=0.70)$ ACCORDING TO THE INCIDENCE ANGLE

\begin{tabular}{|l|r|r|r|r|r|r|r|}
\hline$\theta_{1}\left({ }^{\circ}\right)$ & $\mathbf{7 7}$ & $\mathbf{7 8}$ & $\mathbf{7 9}$ & $\mathbf{8 0}$ & $\mathbf{8 1}$ & $\mathbf{8 2}$ & $\mathbf{8 3}$ \\
\hline$\phi_{R}(\%)$ & 97.2 & 97.7 & 98.1 & 98.5 & 98.9 & 99.2 & 99.4 \\
$\phi_{T}(\%)$ & -69.8 & -72.4 & -75.2 & -78.0 & -80.8 & -83.7 & -86.5 \\
\hline
\end{tabular}

\section{B. Microwave polarizer}

Self-supported graphene ${ }^{1}$ may behave like a polarizer at grazing incidence [14]. This is the case for all frequencies such that the real part of $\sigma$ remains above a few tenths in units of $\varepsilon_{0} c$, typically for $v<50 \mathrm{GHz}$, see Fig. 1. In $p$ polarization, the reflectance $R_{p}$ (eq. 1) remains always smaller than $|\tilde{\sigma} /(2+\tilde{\sigma})|^{2}$ whatever the incidence angle $\theta_{1}$. As $\theta_{1} \rightarrow \pi / 2, R_{p}$ and $A_{p}$ vanish like $\cos ^{2} \theta_{1}$ and $\cos \theta_{1}$, respectively, meaning almost perfect transmittance of graphene at grazing incidence. The reflectance for $s$ polarization takes the same expression as eq. 1 after substitution of the existing sec functions with cos functions. Accordingly, when $n_{1}=n_{2}=1, R_{s}=\left|\tilde{\sigma} /\left(2 \cos \theta_{1}+\tilde{\sigma}\right)\right|^{2}$. Now $R_{p}$ approaches one at grazing incidence. The opposite behaviors of $R_{p}$ and $R_{s}$ for self-supported graphene makes it easy to separate the $s$ and $p$ components of unpolarized microwaves, see Fig. 3. The degree of polarization $\phi=\left(I_{s}-I_{p}\right) /\left(I_{s}+I_{p}\right)$ of the reflected and transmitted beams are listed in table II for different incidence angles $\left(I_{s}\right.$ and $I_{p}$ being the intensities of the $s$ - and $p$-polarized waves).

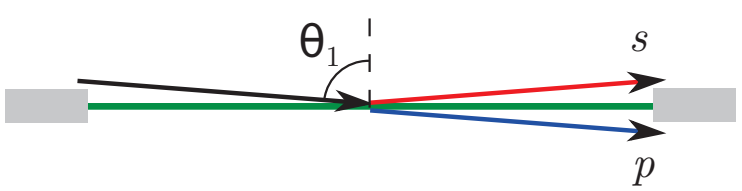

Fig. 3. Unpolarized microwave radiations arriving at grazing incidence on self-supported graphene are separated in reflected and transmitted beams having almost pure $s$ and $p$ polarizations, respectively.

\section{Modification of the Brewster angle}

The Brewster angle $\theta_{B}$ of the interface between two transparent media is modified by an angle $\delta \theta_{1}$ when there is a conducting layer in between [2]. The layer can be a monolayer graphene or a few layer graphene. It suffices then to multiply the conductivity given by eq. 3 by the number of layers [8]. For real $\tilde{\sigma}$, which is the case for graphene for wavelengths $0.4 \mu \mathrm{m}<\lambda<3 \mu \mathrm{m}$, the numerator of eq. 1 is zero at the modified Brewster angle $\theta_{1}=\theta_{B}+\delta \theta_{1}$. The assumed small modification $\delta \theta_{1}$ induces a small change $\delta \theta_{2}$ of the refraction angle $\theta_{2}=\pi / 2-\theta_{B}$. According to Snell's law, $\delta \theta_{2} / \delta \theta_{1}$ $=n_{1} \cos \theta_{B} / n_{2} \cos \left(\pi / 2-\theta_{B}\right)=\left(n_{1} / n_{2}\right) \cot \theta_{B}$. To first order, $\sec \theta_{1}=\sec \theta_{B}\left(1+\tan \theta_{B} \delta \theta_{1}\right)$ and $\sec \theta_{2}=\csc \theta_{B}\left(1+\cot \theta_{B} \delta \theta_{2}\right)$ $=\csc \theta_{B}\left[1+\left(n_{1} / n_{2}\right) \cot ^{2} \theta_{B} \delta \theta_{1}\right]$. Inserting these relations in

${ }^{1}$ In practice, the same holds true for graphene deposited on a non-absorbing medium as long as the thickness of the support remains much smaller than the wavelength. 
the condition $-n_{1} \sec \theta_{1}+n_{2} \sec \theta_{2}+\tilde{\sigma}=0$, knowing that $\tan \theta_{B}=n_{2} / n_{1}$, one obtains

$$
\delta \theta_{1}=\frac{n_{1} n_{2}^{3}}{\left(n_{2}^{2}-n_{1}^{2}\right)\left(n_{2}^{2}+n_{1}^{2}\right)^{3 / 2}} \tilde{\sigma} .
$$

When $n_{2}^{2} \gg n_{1}^{2}$, this expression reproduces eq. 5 of ref. [2]. Eq. 8 is valid when $\tilde{\sigma} \ll\left|n_{2}-n_{1}\right|$. Then, the modification of the Brewster angle is directly proportional to the real part of the reduced conductivity $\tilde{\sigma}$. In the NIR and visible ranges, the angular deviation $\delta \theta_{1}$ can be measured by ellipsometry. For graphene on a thin silica plate, it has been found that $\delta \theta_{1} \approx 0.3^{\circ}$ at $\lambda=600 \mathrm{~nm}$, and this angle correction scales linearly with the number of graphene layers [2]. The application of eq. 8 with $n_{1}=1, n_{2}=1.5$ and $\tilde{\sigma}=\alpha \pi$ gives $\delta \theta_{1}=0.60^{\circ}$. The factor of two compared to the measurements comes from the reflection of light at the back side of the silica plate (see supplementary materials of ref [2]). By measuring $\delta \theta_{1}$ in the visible range, knowing the optical reduced conductivity of monolayer graphene, one can get the actual number of atomic planes contained in the graphene overlayer.

\section{Plasmonics}

The surface plasmon of graphene corresponds to an oscillation of charge localized on the atomic plane, with evanescent fields on both sides. Mathematically, this eigenmode can be created by a vanishingly-small external excitation. The TM plasmon is therefore associated with the zero(s) of the denominator of eqs. 1 or 2 . Taking into account the evanescent character of the "reflected" and "transmitted" waves, the secant functions are purely imaginary variables given by $\sec \theta_{j}=-i\left[\left(k c / \omega n_{j}\right)^{2}-1\right]^{-1 / 2}, j=1,2$, with $k$ the $2 \mathrm{D}$ wave vector of the plasmon. After insertion of this expression in the denominator of eq. 1 , the dispersion relation of the TM plasmon is readily derived in the form [15]

$$
\frac{\epsilon_{1} \omega}{\sqrt{(k c)^{2}-\epsilon_{1} \omega^{2}}}+\frac{\epsilon_{2} \omega}{\sqrt{(k c)^{2}-\epsilon_{2} \omega^{2}}}+i \frac{\sigma}{\varepsilon_{0} c}=0
$$

with $\epsilon_{j}=n_{j}^{2}$ the permittivity of the surrounding media. The existence of a long-lived TM plasmon demands both a vanishing real part of the conductivity $\sigma$ and a positive imaginary part of $\sigma \cdot \operatorname{Im} \sigma>0$ and $\operatorname{Re} \sigma \ll \operatorname{Im} \sigma$ can be realized near the minimum of $\operatorname{Re} \sigma$ in the frequency domain $\gamma<\omega<2 \mu / \hbar$ (see Fig. 1). There are many applications of graphene in plasmonics, most of them require pattering the graphene or depositing a periodic grating on it for exciting the surface plasmon with an external radiation, see ref. [16].

\section{ACKNOWLEDGMENT}

This work has benefited from funding of the H2020 RISE project N 734164 "Graphene-3D". P. K. is thankful for support by Tomsk State University Competitiveness Improvement Program. M. L. thanks the BAEF foundation.

\section{REFERENCES}

[1] Optical properties of graphene L.A. Falkovsky, J. Phys.: Conf. Series 129 (2008) 012004.1-7.

[2] Modied Brewster angle on conducting 2D materials B. Majérus, M. Cormann, N. Reckinger, M. Paillet, L. Henrard, Ph. Lambin, M. Lobet, 2D Mater. 5 (2018) 025007.1-8.

[3] P. Kuzhir et al, to be published.

[4] How effectively do carbon nanotube inclusions contribute to the electromagnetic performance of a composite material? Estimation criteria from microwave and terahertz measurements

M.V. Shuba, D. Yuko, P. Kuzhir, S. Maksimenko, M. Kanygin, A. Okotrub, R. Tenne, and Ph. Lambin, Carbon 129 (2018) 688-94.

[5] Flexible transparent graphene/polymer multilayers for efficient electromagnetic field absorption

K. Batrakov, P. Kuzhir, S. Maksimenko, A. Paddubskaya, S. Voronovich, $\mathrm{Ph}$. Lambin, T. Kaplas, and Yu. Svirko, Scientific Reports 4 (2014) 7191.1-5.

[6] Enhanced microwave-to-terahertz absorption in graphene

K. Batrakov, P. Kuzhir, S. Maksimenko, N. Volynets, S. Voronovich, A. Paddubskaya, G. Valusis, T. Kaplas, Yu. Svirko, and Ph. Lambin, Appl. Phys. Lett. 108 (2016) 123101.1-3.

[7] Low frequency behavior of CVD graphene from dc to $40 \mathrm{GHz}$ R. Wang, S. Raju, M. Chan, and L.J. Jiang, Progr. Electrom. Res. C 71 (2017) 1-7.

[8] Seeing many-body effects in single- and few-layer graphene: observation of two-dimensional saddle-point excitons

K.F. Mak, J. Shan, and T.F. Heinz, Phys. Rev. Lett. 106 (2011) 46401.14.

[9] Microwave absorption on a thin film H. Bosman, Y.Y. Lau, and R.M. Gilgenbach, Appl. Phys. Lett. 82 (2003) 1353-5.

[10] A closed-form approximate expression for the optical conductivity of graphene

E. Simsek, Opt. Lett. 38 (2013) 1437-9.

[11] Perfect electromagnetic absorption using graphene and epsilon-near-zero metamaterials

M. Lobet, B. Majerus, L. Henrard, and Ph. Lambin, Phys. Rev. B 93 (2016) 235424.1-7.

[12] Electrodynamics of graphene/polymer multilayers in the $\mathrm{GHz}$ frequency domain

Ph. Lambin, M. Lobet, K. Batrakov and P. Kuzhir, in "Fundamental and Applied NanoElectroMagnetics", edit. A. Maffucci and S. Maksimenko (NATO Sciences for Peace and Security - Series B, Springer, Amsterdam, 2016), 45-67.

[13] Tunable microwave absorption of graphene-polymer heterostructures deposited on a epsilon-near-zero metamaterial

M. Lobet and M. Sarazzin, in "Proceedings of the 10th International Congress on Advanced Electromagnetic Materials in Microwaves and Optics" (IEEE Inc, 2016) 211-3.

[14] Unusual reflection of electromagnetic radiation from a stack of graphene layers at oblique incidence

Yu.V. Bludov, N.M.R. Peres, and M.I. Vasilevskiy, J. Opt. 15 (2013) 114004.

[15] Plasmonics in graphene at infrared frequencies M. Jablan, H. Buljan, and M. Soljačić, Phys. Rev. B 80 (2009) 245435.17.

[16] Plasmons in graphene: Recent progress and applications X. Luo, T. Qiu, W. Lu, and Z. Ni, Mater. Sci. Engineer R: Reports 74 (2013) 351-76. 